\title{
Light-Cone Formulation of D2-Brane
}

\author{
R. Manvelyan ${ }^{*}$ A. Melikyan, R. Mkrtchyan ${ }^{\dagger}$ \\ Theoretical Physics Department, \\ Yerevan Physics Institute \\ Alikhanyan Br. st.2, Yerevan, 375036 Armenia
}

May 8, 2018

\begin{abstract}
We apply the light-cone hamiltonian approach to D2-brane, and derive the equivalent gauge-invariant Lagrangian. The later appears to be that of three-dimensional Yang-Mills theory, interacting with matter fields, in the special external induced metric, depending on matter fields. The duality between this theory and 11d membrane is shown.
\end{abstract}

\footnotetext{
*E-mail: manvel@moon.yerphi.am

${ }^{\dagger}$ E-mail:mrl@amsun.yerphi.am
} 


\section{Introduction}

The recent developments in the field of higher-dimensional extended objects has led to the deep understanding of the non-perturbative aspects of the superstrings and supergravity theories. That results in the unification, through the notion of duality, of all five different superstrings theories, and, moreover, the new, so-called M-theory [1] notion appeared. The M-theory is intrinsically eleven-dimensional, and contains, in the spectrum of excitations, eleven-dimensional supermembrane theory. Also a new, in comparison with well-known p-branes (extended objects with p space dimensions) extended objects, so called D-branes [2], appeared, which contain, in their spectrum, a vector fields (or, in the case of eleven-dimensional 5-brane - a second rank selfdual tensor field). The main goal of present paper is to investigate the light-cone formalism for the bosonic part of action of D-membranes. The presence of vector field gives a crucial difference from the known case of membranes [3], and leads to an interesting results. The supersymmetrization will be discussed elsewhere.

The light-cone formulation of super-membrane obtained by [3] is closely connected to the Matrix model representation of M-theory [4] . Corresponding bosonic part of area-preserving action, from which we can obtain the Matrix model by replacing Lie brackets by commutators, looks like[3]:

$$
S_{m}=\int d \tau d^{2} \sigma\left[\frac{1}{2}\left(D_{0} X^{M}\right)^{2}-\frac{1}{4}\left\{X^{M}, X^{N}\right\}\left\{X^{M}, X^{N}\right\}\right]
$$

where $M=1,2, \ldots 9$ and $D_{0}=\partial_{0}+\{\omega, \ldots\}$ is a covariant area-preserving derivative with gauge field $\omega\left(\tau, \sigma_{1}, \sigma_{2}\right)$ and Lie bracket

$$
\{X, Y\}=\varepsilon^{i j} \partial_{i} X \partial_{j} Y, \quad i, j, . .=1,2 .
$$

That Lagrangian can be interpreted as a 10-dimensional Yang-Mills theory (if we start from 11-dimensional target space for membrane) reduced to one dimension.

The next new class of extended objects are, as mention above, the socalled D-branes [2]. Main feature of D-branes is that 10-dimensional superstrings can end on them. The incorporation of D-branes in superstring picture gives a lot of issues in the theory of solitonic states in non-perturbative string theory and permits to reveal a different aspects of strings/M-theory dualities. 
In this article we shall investigate the light-cone formulation of the 10dimensional D-membrane described by Dirac-Born-Infeld (DBI) Lagrangian:

$$
\begin{gathered}
L_{D B I}=-\sqrt{-G} ; \quad G=\operatorname{det}\left\{g_{\mu \nu}+F_{\mu \nu}\right\} \\
g_{\mu \nu}=\partial_{\mu} X^{M} \partial_{\nu} X^{M}, \quad F=\partial_{\mu} A_{\nu}-\partial_{\nu} A_{\mu} \\
M=+,-, 1,2, \ldots 8 ; \quad \mu, \nu=0,1,2,
\end{gathered}
$$

which is a bosonic part of D2-brane. We shall construct the analog of areapreserving membrane action (1) for the DBI case.

The main result of our paper is that corresponding gauged light-cone action for D2-brane can be rewritten as a three-dimensional Maxwell theory with matter fields, in the specific curved induced metric:

$$
\tilde{G}_{\mu \nu}=\left(\begin{array}{ll}
-g+\xi^{i}(\omega) g_{i j} \xi^{j}(\omega) & \xi^{k}(\omega) g_{k j} \\
\xi^{k}(\omega) g_{k i} & g_{i j}
\end{array}\right)
$$

where $g_{i j}=\partial_{i} X^{M} \partial_{j} X^{M}, \xi^{i}(\omega)=\varepsilon^{k i} \partial_{k} \omega$ and $g=\operatorname{det} g_{i j}$

The second result is that the duality transformation defined with this metric tensor connects our D2-brane light-cone action to the eleven-dimensional membrane light-cone action (connection between D2-brane in 10d and membrane in 11d was first observed by M. J. Duff and J. X. Lu [5]. Schmidhuber [6] and Townsend [7] established this connection in both directions). In our formulation we start from DBI action and obtain finally some duality transformation in light-cone formulation directly connecting that with membrane light-cone action obtained from Nambu-Goto membrane action in 11 dimension(11) .

\section{Hamiltonian formulation}

Let's start with Hamiltonian formulation for action (3) after preliminary light-cone gauge fixing:

$$
X^{+}\left(\tau, \sigma_{i}\right)=X^{+}(0)+\tau, \quad X^{ \pm}=\sqrt{\frac{1}{2}}\left(X^{10} \pm X^{0}\right)
$$


In this gauge we have the following components of induced metric:

$$
\begin{aligned}
G_{r s} & =g_{r s}+F_{r s}=\partial_{r} X^{M} \partial_{s} X^{M}+\partial_{r} A_{s}-\partial_{s} A_{r}, \\
G_{0 r} & =g_{0 r}+F_{0 r}=\partial_{0} X^{M} \partial_{r} X^{M}+\partial_{r} X^{-}+\partial_{0} A_{r}-\partial_{r} A_{0}, \\
G_{r 0} & =g_{0 r}-F_{0 r}=\partial_{0} X^{M} \partial_{r} X^{M}+\partial_{r} X^{-}+\partial_{r} A_{0}-\partial_{0} A_{r}, \\
G_{00} & =g_{00}=\partial_{0} X^{M} \partial_{0} X^{M}+2 \partial_{0} X^{-} \\
M & =1,2, \ldots 8 ; \quad r, s=1,2
\end{aligned}
$$

The determinant of the induced metric and Lagrangian can be written in the form:

$$
\begin{aligned}
G & =-\Delta \bar{G} ; \quad \Delta=-G_{00}+G_{0 r} G^{r s} G_{s 0} \\
L & =-\sqrt{\Delta \bar{G}}, \bar{G}=\operatorname{det} G_{r s}
\end{aligned}
$$

Then we can write down the canonical momenta:

$$
\begin{aligned}
P^{M} & =\frac{\partial L}{\partial \dot{X}^{M}}=\sqrt{\frac{\bar{G}}{\Delta}}\left[\partial_{0} X^{M}-\frac{1}{2}\left(\partial_{r} X^{M} G^{r s} G_{s 0}+G_{0 r} G^{r s} \partial_{s} X^{M}\right)\right], \\
P^{+} & =\frac{\partial L}{\partial \dot{X}^{-}}=\sqrt{\frac{\bar{G}}{\Delta}}, \quad P_{A}^{r}=\frac{\partial L}{\partial \dot{A}_{r}}=\sqrt{\frac{\bar{G}}{\Delta}}\left[G_{0 s} G^{s r}+G^{r s} G_{s 0}\right]
\end{aligned}
$$

and primary Hamiltonian density and primary constraints:

$$
\begin{aligned}
H & =\frac{P^{M} P^{M}+P_{A}^{r} P_{A}^{s} g_{r s}+\bar{G}}{2 P^{+}}+P_{A}^{r} \partial_{r} A_{0}, \\
P_{A}^{0} & =0, \\
\phi_{r} & =P^{M} \partial_{r} X^{M}+P^{+} \partial_{r} X^{-}+P_{A}^{s} F_{r s}=0 .
\end{aligned}
$$

Then from the requirement of conservation of primary constraints we can obtain secondary (Gauss-low) constraint:

$$
\chi=\partial_{r} P_{A}^{r}=0,
$$

corresponding to $U(1)$ gauge invariance. 
So we can firstly fix the gauge $A_{0}=0$ dropping the second term in (9) and correctly resolve the primary constraint (10). After that we have to add remaining first class constraints (12) and(11) to Hamiltonian with arbitrary Lagrange multipliers $\phi_{r}$ and $\lambda$ :

$$
H=\frac{P^{M} P^{M}+P_{A}^{r} P_{A}^{s} g_{r s}+\bar{G}}{2 P^{+}}+c^{r} \phi_{r}+\lambda \chi
$$

After that we can use $\tau$-dependent reparametrizations of $\sigma^{r}$ :

$$
\sigma^{r} \longrightarrow \sigma^{r}+\xi^{r}\left(\tau, \sigma^{s}\right)
$$

corresponding to the constraints (11), for fixing the following gauge:

$$
\pi_{r}=g_{0 r}+\partial_{0} A_{k} g^{k m} F_{r m}=0,
$$

where the velocities $\dot{A}_{k}$ and $\dot{X}^{M}$ have to be expressed throw corresponding momenta and coordinates. In this and only in this gauge after simple but tedious algebraic calculation one can prove that

$$
\begin{aligned}
c^{r} & =0 \\
\partial_{0} P^{+} & =0
\end{aligned}
$$

according to Hamiltonian equations of motion. It means that in analogy with an ordinary membrane [3] we can put $\left.P^{+}=1\right]$ and express $X^{-}$coordinate through the transversal ones :

$$
\partial_{r} X^{-}=-\left(P^{M} \partial_{r} X^{M}+P_{A}^{s} F_{r s}\right)
$$

It is easy to see that after using of this expression we shall obtain the residual constraint:

$$
\partial_{s} \varepsilon^{s r}\left(P^{M} \partial_{r} X^{M}+P_{A}^{t} F_{r t}\right)=0
$$

Moreover, in that gauge(14) the expressions for momenta look very simple:

\footnotetext{
${ }^{1}$ Strictly speaking we have to put $P^{+}=$const. $\times w\left(\sigma_{i}\right)$, but this leads only to some density factor in definition of Lie bracket [3].
} 


$$
\begin{aligned}
P^{M} & =\partial_{0} X^{M} \\
P_{A}^{r} & =g^{r s} \partial_{0} A_{s}
\end{aligned}
$$

where

$$
g^{r s}=\frac{\varepsilon^{r t} \varepsilon^{s p} g_{t p}}{g}
$$

So, finally we obtain the following expressions for momenta, Hamiltonian and residual constrains in light-cone gauge:

$$
\begin{aligned}
P^{M} & =\partial_{0} X^{M}, \quad P_{A}^{r}=g^{r s} \partial_{0} A_{s} \\
H & =\frac{P^{M} P^{M}+P_{A}^{r} P_{A}^{s} g_{r s}+\bar{G}}{2}, \\
\phi & =\partial_{s} \varepsilon^{s r}\left(P^{M} \partial_{r} X^{M}+P_{A}^{t} F_{r t}\right)=0 \\
\chi & =\partial_{r} P_{A}^{r}=0
\end{aligned}
$$

\section{Gauged Lagrangian}

The main idea of this section is to find out the Lagrangian containing fields $X^{M}, A_{r}$ and two gauge field $\omega\left(\tau, \sigma_{i}\right)$ and $Q\left(\tau, \sigma_{i}\right)$ with the following properties:

1.The expressions (20) and (21) have to be derived as standard expressions of the canonical momenta and Hamiltonian for that Lagrangian in the gauge

$$
\begin{aligned}
& \omega\left(\tau, \sigma_{i}\right)=0 \\
& Q\left(\tau, \sigma_{i}\right)=0
\end{aligned}
$$

2.The equation of motion for gauge fields $\omega\left(\tau, \sigma_{i}\right)$ and $Q\left(\tau, \sigma_{i}\right)$ have to coincide (in the gauge (24)) with corresponding constraints (22) and(23).

3.This Lagrangian has to be gauge invariant with following gauge groups:

a)group of area-preserving diffeomorphisms corresponding to the constraint (22): 


$$
\phi=\left\{\partial_{0} X^{M}, X^{N}\right\}+\varepsilon^{s r} \partial_{s}\left(\partial_{0} A_{k} g^{k t} F_{r t}\right)=0
$$

b)group of $U(1)$ gauge transformations connected to constraint (23):

$$
\chi=\partial_{r}\left(g^{r s} \partial_{0} A_{s}\right)=0
$$

The desired Lagrangian has the following form:

$$
L=\frac{\left(D_{0} X^{M}\right)^{2}}{2}+\frac{1}{2} g^{r s}\left(D_{0} A_{r}-\partial_{r} Q\right)\left(D_{0} A_{s}-\partial_{s} Q\right)-\frac{\bar{G}}{2},
$$

where $\bar{G}=g+F_{12}^{2}$ and

$$
\begin{aligned}
& D_{0} X^{M}=\partial_{0} X^{M}-\varepsilon^{i j} \partial_{i} \omega \partial_{j} X^{M}=\partial_{0} X^{M}-\left\{\omega, X^{M}\right\}=\partial_{0} X^{M}-£_{\xi(\omega)} X^{M} \\
& D_{0} A_{r}=D_{0} A_{r}=\partial_{0} A_{r}-\varepsilon^{i j} \partial_{r} \partial_{i} \omega A_{j}-\varepsilon^{i j} \partial_{i} \omega \partial_{j} A_{r}=\partial_{0} A_{r}-£_{\xi(\omega)} A_{r} .
\end{aligned}
$$

Here $£_{\xi(\omega)}$ is the Lie derivative in direction of divergenceless vector field $\xi^{i}(\omega)=\varepsilon^{k i} \partial_{k} \omega$.

Lagrangian (27) satisfies all three conditions, the gauge transformations for given fields are following:

$$
\begin{aligned}
\delta_{\varepsilon} X^{M} & =\left\{\varepsilon, X^{M}\right\}, \delta_{\varepsilon} A_{r}=£_{\xi(\omega)} A_{r}, \\
\delta_{\varepsilon} Q & =\{\varepsilon, Q\}, \delta_{\varepsilon} \omega=\partial_{0} \varepsilon+\{\varepsilon, \omega\} \\
\delta_{\alpha} X^{M} & =0, \delta_{\alpha} A_{r}=\partial_{r} \alpha, \delta_{\alpha} Q=\partial_{0} \alpha+\{\alpha, \omega\}, \delta_{\alpha} \omega=0 .
\end{aligned}
$$

It is easy to see that $U(1)$ gauge transformations of $Q$ do not commute with area-preserving ones.

This can be improved by redefinition of field $Q$ :

$$
A_{0}=Q+\varepsilon^{i j} \partial_{i} \omega A_{j}
$$

Here we introduce new $A_{0}$ component, which, differently from $Q$, transforms (under area-preserving diffeomorphisms) not as a scalar but as a zero component of three-dimensional vector field : 


$$
\begin{aligned}
& \delta_{\alpha} A_{0}=\partial_{0} \alpha \\
& \delta_{\varepsilon} A_{0}=\left\{\varepsilon, A_{0}\right\}+\partial_{0} \xi^{i}(\varepsilon) A_{i}
\end{aligned}
$$

After that the Lagrangian (27) can be rewritten in the following form:

$$
\begin{aligned}
L & =\frac{\left(D_{0} X^{M}\right)^{2}}{2}-\frac{1}{4}\left\{X^{M}, X^{N}\right\}\left\{X^{M}, X^{N}\right\}+\frac{1}{2} g^{i j} F_{0 i} F_{0 j} \\
& +\frac{1}{2} g^{i j} \xi^{m}(\omega) \xi^{n}(\omega) F_{i m} F_{j n}-\frac{1}{2} F_{12}^{2}+\frac{1}{2} g^{i j} F_{0 i} F_{j n} \xi^{n}(\omega)+g^{i j} \xi^{m}(\omega) F_{i m} F_{0 j}
\end{aligned}
$$

here $F_{0 r}=\partial_{0} A_{r}-\partial_{r} A_{0}$.

Therefore, after introduction of three-dimensional metric $\tilde{G}_{\mu \nu}$ (四) with following properties:

$$
\begin{aligned}
\tilde{G}_{\mu \nu} & =\left(\begin{array}{cc}
-g+\xi^{i}(\omega) g_{i j} \xi^{j}(\omega) & \xi^{k}(\omega) g_{k j} \\
\xi^{k}(\omega) g_{k i} & g_{i j}
\end{array}\right), \\
\tilde{G}^{\mu \nu} & =\left(\begin{array}{cc}
-1 / g & \xi^{j}(\omega) / g \\
\xi^{i}(\omega) / g & g^{i j}-\xi^{i}(\omega) \xi^{j}(\omega) / g
\end{array}\right), \\
g_{i j} & =\partial_{i} X^{M} \partial_{j} X^{M}, g=\operatorname{det} g_{i j}, \xi^{i}(\omega)=\varepsilon^{k i} \partial_{k} \omega \\
\operatorname{det} \tilde{G}_{\mu \nu} & =\tilde{G}, \sqrt{-\tilde{G}}=g, \quad \sqrt{-\tilde{G}} G^{00}=-1
\end{aligned}
$$

and using (19)we can obtain from (33) the final expression for our effective light-cone action:

$$
\begin{aligned}
L= & -\frac{1}{2} \sqrt{-\tilde{G}} \tilde{G}^{\mu \nu} \partial_{\mu} X^{M} \partial_{\nu} X^{M}+\frac{1}{2} \sqrt{-\tilde{G}} \\
& -\frac{1}{4} \sqrt{-\tilde{G}} \tilde{G}^{\mu \nu} \tilde{G}^{\sigma \lambda} F_{\mu \sigma} F_{\nu \lambda}, \\
\partial_{\mu}= & \left(\partial_{0}, \partial_{i}\right), F_{\mu \sigma}=\left(F_{0 r}, F_{i j}=F_{12} \varepsilon_{i j}\right),
\end{aligned}
$$

Here we used relation:

$$
\left\{X^{M}, X^{N}\right\}\left\{X^{M}, X^{N}\right\}=\sqrt{-\tilde{G}}\left(\tilde{G}^{i j}+\xi^{i}(\omega) \xi^{j}(\omega) / g\right) \partial_{i} X^{M} \partial_{j} X^{M}
$$


So, we proved that effective action for light-cone 10d D2-brane can be expressed in the form of usual three-dimensional abelian gauge field coupled to eight scalar matter field $X^{M}$ in the induced metric (34)defined by the same matter fields - target space coordinates $X^{M}$.

\section{Duality transformation}

Let us introduce the new coordinate $X^{9}$ by standard Abelian duality transformation with our metric (34).

For that let us add to (35) metric independent topological term :

$$
\int L(X, \omega, F) d \tau d^{2} \sigma+\frac{1}{2} \int X^{9} d F
$$

Here $F$ is independent second rank antisymmetric tensor field.

Integration over $X^{9}$ leads to (35). But integration over $F$ gives the following equation of motion:

$$
\partial_{\mu} X^{9}=\sqrt{-\tilde{G}} \varepsilon_{\mu \nu \lambda} \tilde{G}^{\nu \rho} \tilde{G}^{\lambda \sigma} F_{\rho \sigma}
$$

or in components:

$$
\begin{aligned}
\partial_{0} X^{9} & =F_{12} \\
\partial_{i} X^{9} & =\varepsilon_{i j}\left(g^{j k}-\xi^{j}(\omega) \xi^{k}(\omega) / g\right) F_{0 k},
\end{aligned}
$$

We see that the substitution of (38) in (36) leads to:

$$
L=\frac{1}{2}\left(D_{0} X^{M}\right)^{2}-\frac{1}{4}\left\{X^{M}, X^{N}\right\}\left\{X^{M}, X^{N}\right\}
$$

where $M, N, .=1,2, \ldots 9$.

This is the light-cone effective Lagrangian for eleven-dimensional membrane. As mentioned above, connection between 10d D2-brane and 11d membrane was established and exploited in [5], [7], [6]. 


\section{Conclusion}

In the present paper the light-cone formalism is developed for 10-dimensional D2-brane, and it was shown, that all corresponding equations of motion and constraints can be derived from the Lagrangian of usual 3d Maxwell theory, interacting with matter fields, in a curved space-time with a special induced metric. This theory is invariant with respect to usual Abelian gauge transformations of gauge fields, and with respect to area-preserving diffeomorphisms. So, we have shown, that complicated non-linear DBI Lagrangian can be substituted, at least at classical level, in light-cone gauge, with quadratic one over gauge fields, although the dependence on a matter fields (coordinates of membrane) remains highly non-linear. There is no any direct connection to a small (gauge) field expansion of initial DBI Lagrangian, although they seem to be similar (but of course there is a lot of differences). The exact integration over gauge fields can be carried out now, at least formally, the corresponding determinant has to be considered as an effective action for Dbrane, and can be expanded by the Riemann tensor (and it's derivatives) of the metric (34). The properties of that tensor, with given special metric, may be very peculiar. Another way of thinking (which was an initial motivation of the present study) is possible connection with Matrix models. Unfortunately, there is no any evident way of interpreting fields in Lagrangian (35) as a matrixes, with corresponding immersion of gauge groups. Nevertheless, there are some indications that literally same Lagrangian can be derived for D3-brane. That problem, together with supersymmetrization of these results will be considered in separate paper 8]

\section{Acknowledgments}

This work was supported in part by the U.S. Civilian Research and Development Foundation under Award \# 96-RP1-253 and by INTAS grants \# 96-538 and \# 93-1038 (ext) . 


\section{References}

[1] E.Witten, Nucl.Phys.B443 (1996)85,

J.Schwarz, "Lectures on superstring and M-theory dualities"

hep-th/9607201.

[2] J.Polchinski, "TASI Lectures on D-Branes", hep-th/961150, J.Polchinski, Phys.Rev.Lett.75 (1995)4724.

[3] B.de Wit, J.Hoppe and H.Nicolai , Nucl.Phys. B305 [FS23](1988)545.

[4] T.Banks, W.Fischler, S.Shenker and L.Susskind, Phys.Rev. D55 (1997)112.

[5] M. J. Duff and J. X. Lu, Nucl. Phys. B390 (1993) 276

[6] C.Schmidhuber, Nucl. Phys. B467 (1996) 146

[7] P.K.Townsend, Phys.Lett. B373 (1996) 68.

[8] R.Manvelyan,A.Melikyan,R.Mkrtchyan, D2 and D3 Branes in light-cone gauge (in preparation). 